\title{
Serum miR-499 as a novel diagnostic and prognostic biomarker in non-small cell lung cancer
}

\author{
MING LI ${ }^{1}$, QIAN ZHANG ${ }^{2}$, LIANG WU ${ }^{3}$, CHENGYOU JIA ${ }^{4}$, FUHUA SHI $^{5}$, SHAOCAI LI ${ }^{6}$, \\ AIMEI PENG ${ }^{1}$, GUOLIANG ZHANG ${ }^{1}$, XIAOLIAN SONG ${ }^{1}$ and CHANGHUI WANG ${ }^{1}$ \\ ${ }^{1}$ Department of Respiratory Medicine, Shanghai 10th People's Hospital, Tongji University, Shanghai 200072; \\ ${ }^{2}$ Department of Nephrology, Huashan Hospital, Fudan University, Shanghai 200032; ${ }^{3}$ Department of Thoracic Surgery, \\ Shanghai Pulmonary Hospital, Tongji University, Shanghai 200433; ${ }^{4}$ Department of Nuclear Medicine, \\ Shanghai 10th People's Hospital, Tongji University, Shanghai 200072; Departments of ${ }^{5}$ Neural Medicine \\ and ${ }^{6}$ Cardiology, People's Hospital of Zunhua County, Tangshan, Hebei 064200, P.R. China
}

Received December 16, 2013; Accepted January 27, 2014

DOI: 10.3892/or.2014.3029

\begin{abstract}
The aim of the present study was to determine whether serum miR-499 may be used as a biomarker for early detection of non-small cell lung cancer (NSCLC). The present study was designed as an initial screening phase and a subsequent validation phase. In the screening phase, we analyzed serum levels of miR-499 in a subset of 40 patients with stage I $(n=20)$ and stage IV $(n=20)$ NSCLC. In the validation phase, miR-499 expression levels in serum $(n=514)$ and tissue $(n=136)$ from NSCLC patients were detected in a large and independent cohort of 514 patients. miR-499 in the screening phase was found to be significantly elevated in the serum of stage I NSCLC patients compared with that in stage IV NSCLC patients $(\mathrm{P}<0.001)$. Validation analysis showed that serum miR-499 levels were robust in differentiating NSCLC patients from control subjects [area under the curve $(\mathrm{AUC})=0.906$; $95 \%$ confidence interval $(\mathrm{CI})=0.879$ to 0.929 ). Serum miR-499 levels were significantly lower in stage III and IV patients compared with those with stage I (both $\mathrm{P}<0.001$ ) or II (both $\mathrm{P}<0.001)$. Low serum miR-499 levels were associated with shorter overall survival and served as an independent prognostic biomarker in NSCLC patients [hazard ratio $(\mathrm{HR})=1.63$; 95\% CI=1.33-2.0; $\mathrm{P}<0.0001$ ). In addition, low serum levels of miR-499 indicated a poor disease-free survival in stage I-II NSCLC patients. Serum miR-499 may prove to be a promising biomarker for early detection and prognosis prediction of NSCLC.
\end{abstract}

Correspondence to: Dr Changhui Wang, Department of Respiratory Medicine, Shanghai 10th People's Hospital, Tongji University, Shanghai 200072, P.R. China

E-mail: wang-changhui@hotmail.com

Key words: lung cancer, microRNA, non-invasive biomarker, early diagnosis, prognostic marker

\section{Introduction}

Lung cancer is the leading cause of cancer-related mortality in both men and women, resulting in 221,130 new cases and 156,940 deaths in the United States in 2011 (1). Non-small cell lung cancer (NSCLC) represents $85 \%$ of all lung cancer cases, and most NSCLC patients present with advanced disease with a very poor rate of cure $(2,3)$. Since the 5 -year survival of patients with metastatic NSCLC is $<15 \%$, prognostic assessment of the patient is essential for the choice of better therapeutic strategies. The current challenge demands the discovery of accurate and non-invasive biomarkers for diagnosis, prognosis and prediction of recurrence to improve the clinical management of NSCLC patients.

During the past decades, several screening tests, including CT scan and bronchoscopy, have been used for early detection of lung cancer (4). However, compliance with these screening tests has been far from adequate. An ideal screening method should have a high sensitivity and specificity for lung cancer. Currently, one of the most important prognostic factors in NSCLC is the anatomical extent of disease, as described by the tumor node metastasis (TNM) classification (5). However, it fails to consider the variety of NSCLC patients, tumor and environmental factors that influence prognosis, and a large variability in disease outcomes that has been observed in subsets of patients with the same clinical features (6). Therefore, there is a clear need to identify biomarkers for the early detection of NSCLC, and to categorize different prognostic groups and improve the clinical management of NSCLC patients.

MicroRNAs (miRNAs) are non-coding RNA molecules of 21-23 nucleotides in length that play an important role in regulation of mRNA expression (7). miRNAs are known to be involved in various cellular processes and are associated with various diseases including cancer $(8,9)$. Several studies have demonstrated that miRNAs play important roles in the initiation and progression of cancer. In addition, miRNA expression profiles and specific miRNAs have been shown to be potential diagnostic or prognostic tools for cancer (10-12). 
Recent studies $(13,14)$ have also shown that circulating miRNAs may constitute accurate methods for diagnosis and prognosis of NSCLC. A study by Markou et al (15) demonstrated that high expression of serum miR-21 and miR-30e-5p was associated with shorter overall survival (OS). Hu et al (14) also reported a 4-miRNA signature (miR-486, miR-30d, miR-1 and miR-499) that predicted survival of stage I-IIIa NSCLC. However, whether the expression profile of circulating miR-499 reflects the miRNA profile of tumor tissue remains unclear. In addition, the relationship between dysregulated miRNAs and tumor stage is also uncertain. In the present study, we evaluated the possible association of miR-499 with early detection of NSCLC and survival of NSCLC patients in an attempt to further clarify the impact of miRNAs on the diagnosis and prognosis of lung cancer.

\section{Materials and methods}

Study design. The present study was approved by the institutional review board of Shanghai Pulmonary Disease Hospital. All participants provided written consent and indicated willingness to donate their blood and tissue samples for research. A total of 568 subjects were enrolled in this study, including 514 NSCLC patients and 54 age- and gender-matched healthy volunteers as controls. NSCLC patients were recruited at Shanghai Pulmonary Disease Hospital affiliated to Tongji University in Shanghai, China, between January 2007 and December 2011. Patients were excluded if they had any of the following: selfreported previous cancer history, metastasis from other organs, or if they underwent chemotherapy or radiotherapy before blood collection. They were followed-up at 3-month intervals by telephone after the first visit to the hospital.

The present study was designed as an initial screening phase and a subsequent validation phase. In the screening phase, serum levels of miR-499 $(14,16)$ were analyzed in a subset of 40 patients with stage I $(n=20)$ and stage IV $(n=20)$ NSCLC. To further assess the specificity of miRNA expression, serum samples were collected from 12 patients with NSCLC and 12 gender- and age-matched normal controls. In the validation phase, miR-499 expression levels in serum $(n=514)$ and tissues $(n=136)$ from NSCLC patients were evaluated in a large and independent cohort of 514 patients.

RNA isolation and qRT-PCR from serum and tissues. miRNAs were extracted from blood samples using the Qiagen miRNeasy kit (Qiagen, Valencia, CA, USA). Briefly, $250 \mu \mathrm{l}$ serum was centrifuged at $10,000 \mathrm{rpm}$ for $10 \mathrm{~min}$ at $4^{\circ} \mathrm{C}$. miRNAs were enriched and purified according to the manufacturer's protocol. To allow for normalization of sample-to-sample variation in the RNA isolation step, synthetic C. elegans miRNA (cel-miR-39) was added to each sample $(12,17)$.

MiRNAs were extracted from fresh frozen tissue samples using PureLink ${ }^{\mathrm{TM}}$ miRNA Isolation kit (Life Technologies Corporation, Carlsbad, CA, USA). Briefly, fresh frozen tissue samples were microdissected to enrich for neoplastic cells. Homogenized samples were centrifuged, followed by deparaffinization and RNA extraction using the manufacturer's protocol.

PCR reactions for quantifying miR-499, miR-39 and miR-16 were performed in triplicate using the TaqMan
microRNA Reverse Transcription kit (Applied Biosystems, USA). qRT-PCR was performed in an ABI Prism 7000 sequence detection system (Applied Biosystems) according to the manufacturer's instructions, with the following cycling conditions: $95^{\circ} \mathrm{C}$ for $10 \mathrm{~min}$, followed by 40 cycles at $95^{\circ} \mathrm{C}$ for $15 \mathrm{sec}$ and $60^{\circ} \mathrm{C}$ for $1 \mathrm{~min}$.

Calculation of miRNA expression. Relative quantification of miR-499 expression was calculated with the $2^{-\Delta \Delta C t}$ method (18) [Applied Biosystems; User Bulletin no. 2 (P/N 4303859)] and normalized using cel-miR-39 (for serum samples) and miR-16 (19) (for tissue samples) using the $2^{-\Delta \Delta C t}$ method, knowing that it facilitates detecting and quantifies exclusively mature miRNAs but not their precursors.

Statistical analysis. Mann-Whitney U and Kruskal-Wallis analyses of variance were used to evaluate statistical differences in serum or tissue miRNA expression between unpaired groups and multiple comparison groups. $\chi^{2}$ test was used for categorical data. A multivariable logistic regression model was used to calculate odds ratios (ORs) for patients associated with NSCLC according to serum miRNA levels.

Disease-free survival (DFS) and OS were measured for each patient. Survival curves were estimated using the Kaplan-Meier method, and differences between them were evaluated by the log-rank test. Cox proportional hazard regression test was used to estimate univariate and multivariate hazard ratios for recurrence and prognosis with a step-down method.

Receiver operating characteristic (ROC) curves were established for discriminating NSCLC and controls. The optimal miRNA expression cut-off threshold values were determined at the point on the ROC curve at which Youden's index (20) was maximal.

All $\mathrm{P}$-values are two-sided and $\mathrm{P}<0.05$ was considered to indicate a statistically significant difference. All statistical analyses were carried out using MedCalc version 11.2 (Mariakerke, Belgium).

\section{Results}

Serum and tissue miR-499 expression during the screening phase. In the screening phase, we investigated the relative expression levels of the miR-499 in a subset of serum specimens from 20 NSCLC patients with stage IV compared with 20 patients with stage I. It was found that miR-499 was significantly elevated in the serum of NSCLC patients with stage I compared with stage IV $(\mathrm{P}<0.001$; Fig. 1A). We next examined the feasibility of detecting the expression of serum miR-499 in 12 NSCLC patients and 12 healthy control subjects. It was found that serum miR-499 levels were significantly decreased in the sera of NSCLC patients $(\mathrm{P}<0.001$; Fig. 1B). Expression of miR-499 was determined in a small set of 12 NSCLCs compared with the adjacent normal tissue. miR-499 level was also found to be reduced in NSCLC tissues compared with normal tissues $(\mathrm{P}<0.001$; Fig. 1C). These results indicated that serum miR-499 was downregulated in NSCLC patients compared with adjacent tissues.

Based on these observations, we focused the rest of our study on miR-499 for further assessment of its efficacy as a 

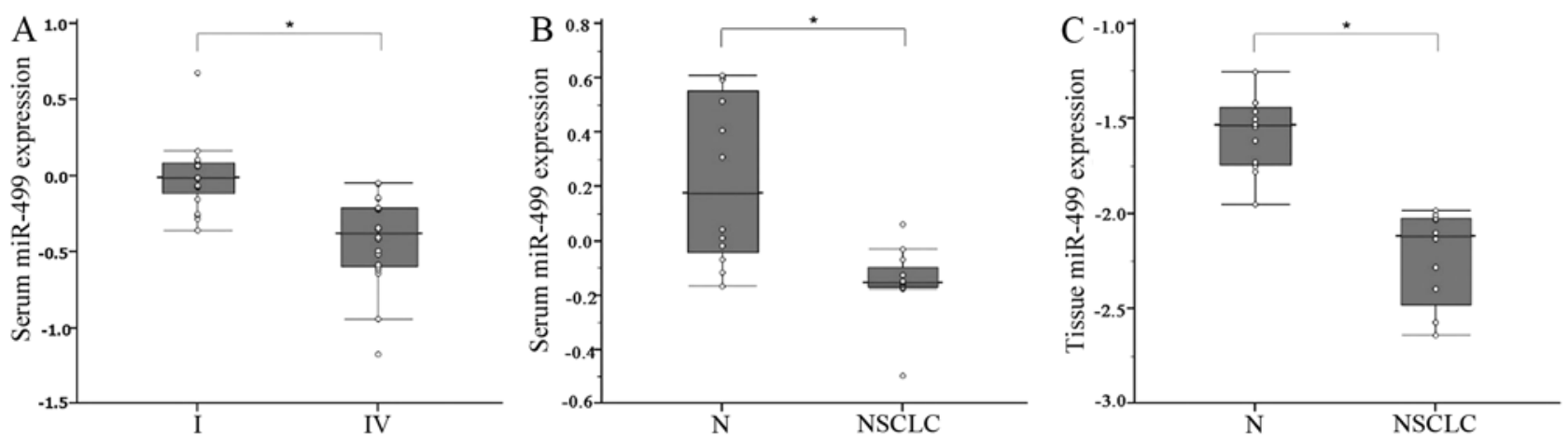

Figure 1. Analysis of miR-499 expression in NSCLC patients and normal controls (N). (A) Box plots of serum levels of miR-499 in patients with stage $\mathrm{I}(\mathrm{n}=20)$ and stage IV ( $n=20)$ NSCLC. (B) Box plots are shown for serum levels of miR-499 from N subjects $(n=12)$ and NSCLC patients $(n=12)$. (C) Box plots are shown for miR-499 levels in primary tumor tissues (NSCLC, $n=12$ ) and the adjacent normal tissues $(n=12)$. Expression levels of miR-499 (log 10 scale at $y$-axis) were normalized to cel-miR-39 and miR-16 expression in sera and tissue. Boxes represent interquartile range, and the horizontal line across each box indicates median value. Mann-Whitney U test was used to determine statistical significance. " $\mathrm{P}<0.001$. NSCLC, non-small cell lung cancer.
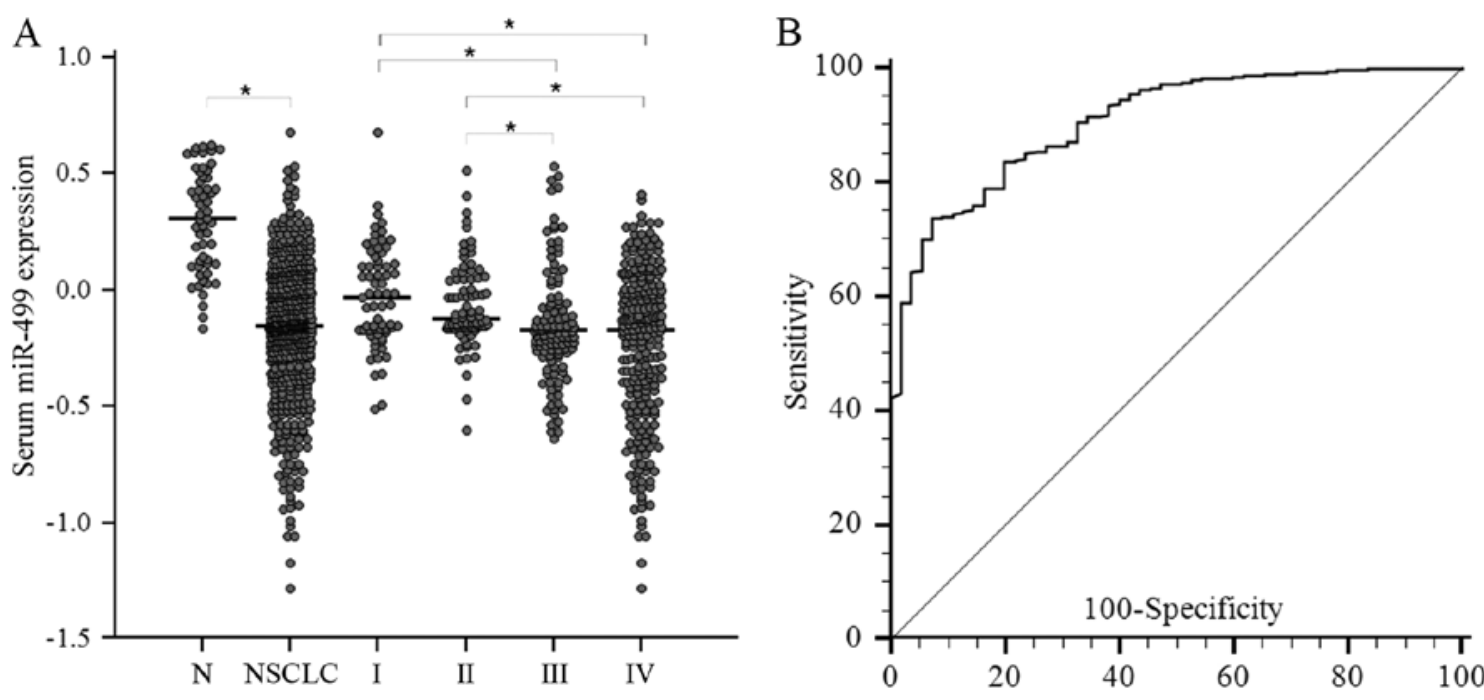

Figure 2. (A) miR-499 expression levels in serum samples. Box plots represent serum miR-499 levels in healthy control subjects (n=54) and different tumor node metastasis (TNM) stages (I, II, III and IV) of NSCLC ( $\mathrm{n}=514)$. The $\mathrm{y}$-axis (log 10 scale) represents relative expression of miR-499 normalized to cel-miR-39. Horizontal line indicates median value. Mann-Whitney U and Kruskal-Wallis tests were used to determine statistical significance. "P<0.001. (B) Receiver operating characteristics (ROC) curve analysis using serum miR-499 for differentiating NSCLC patients from normal control (N) subjects. Serum miR-499 yielded an area under the curve (AUC) value of 0.906 [95\% confidence interval (CI) $=0.879$ to 0.929$]$, with $73.7 \%$ sensitivity and $92.7 \%$ specificity in differentiating NSCLC from normal control subjects. NSCLC, non-small cell lung cancer.

diagnostic, prognostic and recurrence predictive biomarker in NSCLC patients.

Serum miR-499c expression level as a diagnostic biomarker in NSCLC patients. To examine whether miR-499 had diagnostic potential, 568 serum samples, including 514 from NSCLC patients and 54 from normal controls, were analyzed. It was found that miR-499 expression levels were downregulated markedly in sera of NSCLC patients as compared with those in normal controls $(\mathrm{P}<0.001$; Fig. 2A). In addition, when all NSCLC patients were grouped based on TNM stage, miR-499 expression levels were significantly lower in stage III and IV patients than those in stage I or II patients (both $\mathrm{P}<001$, Fig. 2A). The potential clinical significance of serum miR-499 expression is presented in Table I.

Next, we determined whether miR-499 possessed any significance in sensitivity and specificity in lung cancer patients. ROC curves were analyzed, indicating that serum miR-499 levels were robust in differentiating patients with NSCLC from control subjects with an area under the ROC curve (AUC) value of 0.906 (95\% CI=0.879 to 0.929) (Fig. 2B). At the cut-off value of 1.330 for miR-499, the optimal sensitivity and specificity were 73.7 and $92.7 \%$, respectively. Multivariate logistic regression analyses on variables including age, gender and serum miRNAs showed that miR-499 was a potential biomarker for NSCLC diagnosis $(\mathrm{P}<0.0001)$. The OR for patients with miR-499 $<1.0247$ associated with NSCLC was 64.1 (95\% CI: 20.83-197.49). These results indicated that serum miR-499 had potential significance with respect to the sensitivity and specificity in the diagnosis of NSCLC.

Correlation between serum/tissue miR-499 expression and survival of NSCLC patients. Based on the above findings, we further evaluated whether serum miR-499 levels may 
Table I. Association between miR-499 expression in serum and tissue specimens from NSCLC patients and various clinicopathological characteristics.

\begin{tabular}{|c|c|c|c|c|c|c|}
\hline \multirow[b]{2}{*}{ Factors } & \multicolumn{3}{|c|}{ Serum miR-499 (n=514) } & \multicolumn{3}{|c|}{ Tissue miR-499 $(n=136)$} \\
\hline & High $(n=257)$ & Low $(n=257)$ & P-value & High $(n=68)$ & Low $(\mathrm{n}=68)$ & P-value \\
\hline Age (years) & & & 0.28 & & & 0.36 \\
\hline$\leq 65$ & 111 & 98 & & 20 & 26 & \\
\hline$>65$ & 146 & 159 & & 48 & 42 & \\
\hline Gender & & & 0.79 & & & 0.17 \\
\hline Male & 133 & 137 & & 39 & 30 & \\
\hline Female & 124 & 120 & & 29 & 38 & \\
\hline Smoking status & & & 0.36 & & & 0.39 \\
\hline Non-smoker & 105 & 94 & & 34 & 40 & \\
\hline Ever-smoker & 152 & 163 & & 34 & 28 & \\
\hline Stage & & & 0.28 & & & 0.22 \\
\hline I & 34 & 26 & & 28 & 22 & \\
\hline II & 57 & 45 & & 35 & 44 & \\
\hline III & 46 & 56 & & 5 & 2 & \\
\hline IV & 120 & 130 & & 0 & 0 & \\
\hline Histologic type & & & 0.81 & & & 0.016 \\
\hline Adenocarcinoma & 140 & 146 & & 52 & 40 & \\
\hline Squamous cell & 99 & 92 & & 14 & 28 & \\
\hline Others & 18 & 19 & & 2 & 0 & \\
\hline Surgical operation & & & 0.02 & & & 0.93 \\
\hline No & 172 & 196 & & 0 & 0 & \\
\hline Yes & 85 & 61 & & 68 & 68 & \\
\hline Chemotherapy or radiotherapy & & & 0.08 & & & 0.12 \\
\hline No & 55 & 39 & & 5 & 12 & \\
\hline Yes & 202 & 218 & & 63 & 56 & \\
\hline
\end{tabular}

NSCLC, non-small cell lung cancer.

predict the prognosis of NSCLC patients. As anticipated, OS was poorer in patients with lower serum levels of miR-499 $(\mathrm{P}=0.005, \log$-rank test; Fig. 3A). In addition, lower serum levels of miR-499 also predicted poorer survival in patients with stage I-II and III-IV NSCLC $(\mathrm{P}=0.0012$ and $<0.0001$, log-rank test; Fig. 3B and C). However, tissue miR-499 levels were not significantly associated with survival in NSCLC patients ( $\mathrm{P}=0.3139$, log-rank test; Fig. 3D) (Table II). Furthermore, the Cox proportional hazard regression model was used to clarify whether serum miR-499 expression was an independent risk factor for prognosis. The result of univariate analysis showed that poor prognosis in NSCLC patients was significantly associated with low serum miR-499 levels but not with tissue miR-499 levels $(\mathrm{P}=0.0005$ and $\mathrm{P}=0.3139$, respectively), high $\mathrm{T}$ stage (T3/4, $\mathrm{P}<0.0001)$, lymph node metastasis $(\mathrm{N} 1 / 2 / 3, \mathrm{P}<0.0001)$, distant metastasis ( $\mathrm{M} 1, \mathrm{P}<0.0001)$, and high TNM stage (III/IV, $\mathrm{P}<0.0001)$ (Table II). The result of multivariate analysis showed that downregulation of serum miR-499 expression was an independent prognostic marker for predicting poorer OS in NSCLC patients $(\mathrm{HR}=1.63$, 95\% CI=1.33-2.0, $\mathrm{P}<0.0001$; Table II). These results indicated that downregulation of serum miR-499 expression was an independent prognostic marker for predicting poorer OS in NSCLC patients.

Serum miR-499 as a predictive biomarker of tumor recurrence in NSCLC. Next, we analyzed DFS. Patients with low serum miR-499 in stage I-II had shorter DFS $(\mathrm{P}=0.045$, log-rank test). To further evaluate whether serum miR-499 levels may be used as a predictor of tumor recurrence after surgery (stage I-II), the Cox proportional hazard regression model was performed (Table III). The result of univariate analysis showed that poor DFS was significantly associated with low serum levels of miR-499 ( $\mathrm{P}=0.0304)$, high TNM stage (II, $\mathrm{P}<0.0001$ ), and no chemotherapy or radiotherapy $(\mathrm{P}<0.0001)$. The result of multivariate analysis showed that low serum miR-499 expression was an independent predictor for tumor recurrence in patients with stage I-II NSCLC $(\mathrm{HR}=1.96,95 \% \mathrm{CI}=1.03-3.73, \mathrm{P}=0.04)$. These results indicated that serum miR-499 levels may serve not only as a diagnostic and prognostic marker, but also as a predictor of early recurrence of NSCLC as well. 
Table II. Univariate and multivariate analyses for prognostic factors in patients with NSCLC.

\begin{tabular}{|c|c|c|c|c|c|c|}
\hline \multirow[b]{2}{*}{ Variables } & \multicolumn{3}{|c|}{ Univariate } & \multicolumn{3}{|c|}{ Multivariate } \\
\hline & HR & $95 \% \mathrm{CI}$ & P-value & HR & $95 \% \mathrm{CI}$ & P-value \\
\hline Age, years (>65 vs. $\leq 65)$ & 0.84 & $0.61-1.16$ & 0.2855 & & & \\
\hline Gender (female vs. male) & 0.98 & $0.80-1.20$ & 0.8212 & - & - & - \\
\hline Histology (squamous vs. non-squamous) & 0.91 & $0.74-1.12$ & 0.37 & - & - & - \\
\hline Pathological T (T3/4 vs. T1/2) & 2.39 & $1.94-2.94$ & $<0.0001$ & 1.45 & $1.10-1.89$ & 0.0077 \\
\hline Lymph node metastasis (N1-3 vs. N0) & 1.80 & $1.46-2.20$ & $<0.0001$ & 1.21 & $0.95-1.54$ & 0.1333 \\
\hline Distant metastasis (M1 vs. M0) & 2.67 & $2.14-3.32$ & $<0.0001$ & 1.68 & $1.32-2.14$ & $<0.0001$ \\
\hline TNM stage (III/IV vs. I/II) & 3.57 & $2.91-4.40$ & $<0.0001$ & 21.63 & $2.98-156.85$ & 0.0025 \\
\hline Chemotherapy or radiotherapy (no vs. yes) & 1.49 & $0.94-2.38$ & 0.0928 & - & - & - \\
\hline miR-499 in serum (low vs. high) & 1.42 & $1.16-1.75$ & 0.0005 & 1.63 & $1.33-2.00$ & $<0.0001$ \\
\hline miR-499 in tumor (low vs. high) & 1.31 & $0.77-2.21$ & 0.3139 & - & - & - \\
\hline
\end{tabular}

HR, hazard ratio; CI, confidence interval. Cut-off values of miR-499 in tissue and serum are derived from receiver operating characteristic curve with Youden's index. NSCLC, non-small cell lung cancer.
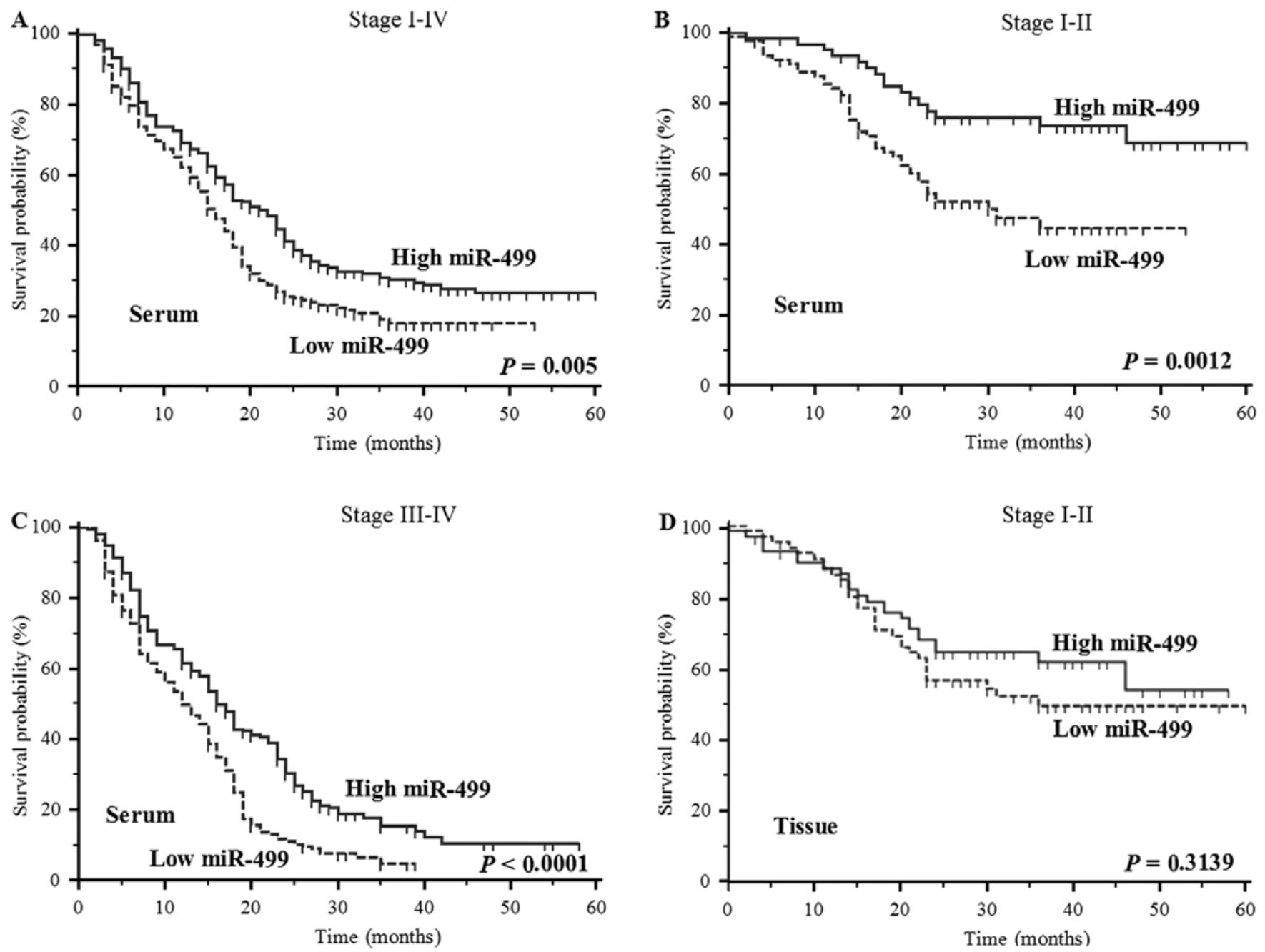

Figure 3. Kaplan-Meier survival curves of NSCLC patients subdivided by miR-499 levels in serum and matched primary tumors from NSCLC patients. Overall survival rates of patients with (A) stage I-IV, (B) stage I-II and (C) stage III-IV NSCLC with low serum miR-200c levels were significantly lower than those with high miR-499 expression (stage I-IV, $\mathrm{P}=0.005$; stage I-II, $\mathrm{P}=0.0012$; stage III-IV, $\mathrm{P}<0.001$, log-rank test). (D) However, miR-499 levels in tissues were not significantly associated with survival in NSCLC patients $(\mathrm{P}=0.3139$, log-rank test). The optimal miRNA expression cut-off threshold values were deduced from the ROC curves with Youden's index. NSCLC, non-small cell lung cancer. 
Table III. Univariate and multivariate analyses for predictive factors of recurrence in patients with stage I-II NSCLC.

\begin{tabular}{|c|c|c|c|c|c|c|}
\hline \multirow[b]{2}{*}{ Variables } & \multicolumn{3}{|c|}{ Univariate } & \multicolumn{3}{|c|}{ Multivariate } \\
\hline & HR & $95 \% \mathrm{CI}$ & P-value & HR & $95 \% \mathrm{CI}$ & P-value \\
\hline Age, years (>65 vs. $\leq 65)$ & 1.11 & $0.62-1.97$ & 0.7346 & & & \\
\hline Gender (female vs. male) & 0.66 & $0.31-1.42$ & 0.29 & - & - & - \\
\hline Pathological T (T2/3 vs. T1) & 2.22 & $0.62-8.01$ & 0.22 & - & - & - \\
\hline Lymph node metastasis (N1 vs. N0) & 1.24 & $0.23-6.69$ & 0.8024 & - & - & - \\
\hline TNM stage (II vs. I) & 2.54 & $1.13-5.71$ & 0.024 & 2.76 & $1.32-5.77$ & 0.007 \\
\hline Chemotherapy or radiotherapy (no vs. yes) & 1.39 & $1.06-1.81$ & 0.0158 & 1.61 & $1.15-2.26$ & 0.0057 \\
\hline miR-499 in serum (low vs. high) & 3.03 & $1.11-8.27$ & 0.0304 & 1.96 & $1.03-3.73$ & 0.04 \\
\hline
\end{tabular}

HR, hazard ratio; CI, confidence interval. Cut-off value of serum miR-499 is derived from receiver operating characteristic curve with Youden's index. NSCLC, non-small cell lung cancer.

\section{Discussion}

In the present study, we investigated the potential clinical utility of serum miR-499 and found that serum miR-499 could serve as a non-invasive biomarker for the diagnosis and prediction of prognosis and tumor recurrence in NSCLC patients. The miR-499 levels in serum samples from NSCLC patients were significantly lower than those in healthy controls. miR-499 had a significant diagnostic value for NSCLC and yielded AUC of 0.906 with $73.7 \%$ sensitivity and $92.7 \%$ specificity in discriminating NSCLC from normal controls. In addition, the OR for case subjects with low levels of miR-499 expression associated with NSCLC was 64.1 (95\% CI; 20.83-197.49), indicating that miR-499 expression may be exploited as a promising non-invasive biomarker for early detection of NSCLC.

Our study also strongly suggested that serum miR-499 expression may serve as a prognostic biomarker for NSCLC. We found that serum miR-499 levels were significantly lower in patients with stage III or IV NSCLC than those in stage I or II NSCLC. In addition, low serum miR-499 levels (rather than tissue) were associated with shorter OS and may prove to be an independent prognostic biomarker in NSCLC patients. In addition, low serum levels of miR-499 expression indicate a poor DFS in stage I-II NSCLC. The multivariable Cox proportional hazards model showed that low serum miR-499 expression was an independent predictor of tumor recurrence in patients with stage I-II NSCLC.

To the best of our knowledge, this is the first report to demonstrate the potential role of serum miR-499 in the early detection of NSCLC. Although the observation of the prognostic value of miR-499 is similar to a previous report (14), our results are the first to demonstrate that low serum miR-499 expression is associated with advanced TNM stage and poor DFS.

Several studies $(21,22)$ have reported that miRNAs are potential diagnostic biomarkers and prognostic factors in lung cancer. In 2004, lung tumor-derived miRNAs were first described in tissue by Takamizawa et al (23), who reported that reduced let-7 expression was significantly associated with shortened postoperative survival. A study by Yanaihara et al (21) showed that the tissue miRNA expression profile was a diagnostic and prognostic marker of lung cancer. Serum miRNAs are resistant to RNase digestion, suggesting that miRNAs in serum are sufficiently stable to serve as a clinical biomarker (12). miR-21 was the first serum miRNA biomarker discovered by Lawrie et al (24), who reported that high serum levels of miR-21 in patients with diffuse large B cell lymphoma were associated with increased relapse-free survival. Chen et al (9) also demonstrated that serum let-7 level was increased in lung cancer patients as compared with healthy controls. In addition, serum miRNAs are prognostic factors for lung cancer. Hu et al (14) found that serum levels of 4 miRNAs (miR-486, miR-30d, miR-1 and miR-499) were significantly associated with OS of NSCLC patients. Boeri et al (25) described plasma miRNA signatures for high risk of lung cancer, diagnosis and prognosis. These findings indicate that circulating miRNAs may be noninvasive diagnostic or prognostic markers for lung cancer.

Previous studies usually focused on the association between miR-499 and the muscle and heart. Wang et al (26) detected the presence of plasma miR-499 in patients with acute myocardial infarction with very low abundance, suggesting it may be a biomarker for early detection of myocardial injury. Donaldson et al (27) found that miR-499 was elevated in the plasma of COPD patients as compared with controls. Recently, Vinci et al (16) found that the expression of miR-499 in NSCLC tissues tended to be lower than controls but the reduction was not statistically significant $(\mathrm{P}=0.123)$. However, they found that increased expression of miR-499 was associated high tumor grade. Hu et al (14) showed that low serum levels of miR-499 were significantly associated with short OS. Therefore, the clinical significance of circulating miR-499 levels in NSCLC remains unclear.

The present study demonstrated that the relative expression of serum miR-499 was significantly different between NSCLC patients and normal controls, suggesting that serum miR-499 level may be a useful biomarker for the clinical diagnosis of NSCLC. Screening for lung cancer has long been of interest, with the hope of reducing the number of patients diagnosed with advanced disease. Although screening with low-dose computed tomography (LDCT) has substantially reduced the risk of mortality due to lung cancer, there is a significant chance of a false-positive result $(28,29)$. Therefore, serum miR-499 level with high specificity for early detection of NSCLC may 
be a powerful adjunct to LDCT. Our study showed that preoperative serum miR-499 expression was an independent factor for detecting early tumor recurrence in patients with stage I-II NSCLC. In clinical practice, these patients can significantly benefit from timely clinical intervention, thus improving cancer survival. Our data also support the use of miR-499 as a non-invasive biomarker that can facilitate disease risk assessment, severity and survival time in NSCLC patients.

Although our study suggests that miR-499 is a promising screening and assessment tool for NSCLC, there are potential limitations in using miR-499 as a diagnostic, prognostic and recurrence predictive biomarker. Firstly, as no consensus internal controls for circulating miRNA have been established, we used the ideal approach to normalize experimental miRNA data using spiked-in synthetic, non-human mature miRNA from Caenorhabditis elegans. Although cel-miR-39 had given consistent expression across all patients and controls, and the method of quantifying relative expression of serum miRNAs was widely recognized, absolute quantization of serum miR-499 expression may further improve the translation of these data into clinical application. Secondly, although selection criteria for lung cancer screening were uncertain, it is unclear whether high-risk people benefit from serum miRNAs tests. Plasma miRNA tests may be more functional for noninvasive diagnosis of lung cancer in individuals with solitary pulmonary nodules $(30,31)$. Thirdly, as serum expression of miR-499 has been described in other diseases including COPD and AMI, it may be difficult to differentiate whether serum miR-499 expression is specifically associated with NSCLC. Finally, it is difficult to ensure that the control subjects are healthy, and our clinical materials are solely from Chinese people. A large sample size including diverse ethnic populations may be helpful to eliminate potential sampling error.

In conclusion, serum miR-499 appears to be a novel diagnostic, prognostic and predictive biomarker in patients with NSCLC. Nevertheless, large prospective studies are required to further evaluate our theory before serum miR-499 can be incorporated into routine clinical practice.

\section{Acknowledgements}

This study was supported by the National Natural Science Foundation of China $(81172229,81372175)$ and the China Postdoctoral Science Foundation (First Class, 2013M530212).

\section{References}

1. Siegel R, Ward E, Brawley O and Jemal A: Cancer statistics, 2011: the impact of eliminating socioeconomic and racial disparities on premature cancer deaths. CA Cancer J Clin 61: 212-236, 2011

2. Parkin DM: Global cancer statistics in the year 2000. Lancet Oncol 2: 533-543, 2001

3. Li M, Zhang Q, Fu P, et al: Pemetrexed plus platinum as the first-line treatment option for advanced non-small cell lung cancer: a meta-analysis of randomized controlled trials. PLoS One 7: e37229, 2012.

4. Smith RA, Brooks D, Cokkinides V, et al: Cancer screening in the United States, 2013: a review of current American Cancer Society guidelines, current issues in cancer screening, and new guidance on cervical cancer screening and lung cancer screening. CA Cancer J Clin 63: 88-105, 2013.

5. Goldstraw P, Ball D, Jett JR, et al: Non-small-cell lung cancer. Lancet 378: 1727-1740, 2011.

6. Rami-Porta R and Goldstraw P: Strength and weakness of the new TNM classification for lung cancer. Eur Respir J 36: 237-239, 2010
7. Filipowicz W, Bhattacharyya SN and Sonenberg N: Mechanisms of post-transcriptional regulation by microRNAs: are the answers in sight? Nat Rev Genet 9: 102-114, 2008.

8. van Kouwenhove M, Kedde M and Agami R: MicroRNA regulation by RNA-binding proteins and its implications for cancer. Nat Rev Cancer 11: 644-656, 2011.

9. Chen X, Ba Y, Ma L, et al: Characterization of microRNAs in serum: a novel class of biomarkers for diagnosis of cancer and other diseases. Cell Res 18: 997-1006, 2008.

10. Kong YW, Ferland-McCollough D, Jackson TJ and Bushell M: microRNAs in cancer management. Lancet Oncol 13: e249-e258, 2012.

11. Lu J, Getz G, Miska EA, et al: MicroRNA expression profiles classify human cancers. Nature 435: 834-838, 2005.

12. Mitchell PS, Parkin RK, Kroh EM, et al: Circulating microRNAs as stable blood-based markers for cancer detection. Proc Natl Acad Sci USA 105: 10513-10518, 2008.

13. Voortman J, Goto A, Mendiboure J, et al: MicroRNA expression and clinical outcomes in patients treated with adjuvant chemotherapy after complete resection of non-small cell lung carcinoma. Cancer Res 70: 8288-8298, 2010.

14. Hu Z, Chen X, Zhao Y, et al: Serum microRNA signatures identified in a genome-wide serum microRNA expression profiling predict survival of non-small-cell lung cancer. J Clin Oncol 28: 1721-1726, 2010

15. Markou A, Sourvinou I, Vorkas PA, et al: Clinical evaluation of microRNA expression profiling in non small cell lung cancer. Lung Cancer 81: 388-396, 2013.

16. Vinci S, Gelmini S, Pratesi N, et al: Genetic variants in miR-146a, $m i R-149, m i R-196 a 2, m i R-499$ and their influence on relative expression in lung cancers. Clin Chem Lab Med 49: 2073-2080, 2011.

17. Kroh EM, Parkin RK, Mitchell PS and Tewari M: Analysis of circulating microRNA biomarkers in plasma and serum using quantitative reverse transcription-PCR (qRT-PCR). Methods 50: 298-301, 2010.

18. Livak KJ and Schmittgen TD: Analysis of relative gene expression data using real-time quantitative PCR and the $2^{-\Delta \Delta C_{\mathrm{T}}}$ method. Methods 25: 402-408, 2001.

19. Peltier HJ and Latham GJ: Normalization of microRNA expression levels in quantitative RT-PCR assays: identification of suitable reference RNA targets in normal and cancerous human solid tissues. RNA 14: 844-852, 2008.

20. Ruopp MD, Perkins NJ, Whitcomb BW and Schisterman EF: Youden Index and optimal cut-point estimated from observations affected by a lower limit of detection. Biom J 50: 419-430, 2008.

21. Yanaihara N, Caplen N, Bowman E, et al: Unique microRNA molecular profiles in lung cancer diagnosis and prognosis. Cancer Cell 9: 189-198, 2006.

22. Yu SL, Chen HY, Chang GC, et al: MicroRNA signature predicts survival and relapse in lung cancer. Cancer Cell 13: 48-57, 2008.

23. Takamizawa J, Konishi H, Yanagisawa K, et al: Reduced expression of the let-7 microRNAs in human lung cancers in association with shortened postoperative survival. Cancer Res 64: 3753-3756, 2004.

24. Lawrie CH, Gal S, Dunlop HM, et al: Detection of elevated levels of tumour-associated microRNAs in serum of patients with diffuse large B-cell lymphoma. Br J Haematol 141: 672-675, 2008.

25. Boeri M, Verri C, Conte D, et al: MicroRNA signatures in tissues and plasma predict development and prognosis of computed tomography detected lung cancer. Proc Natl Acad Sci USA 108: 3713-3718, 2011.

26. Wang GK, Zhu JQ, Zhang JT, et al: Circulating microRNA: a novel potential biomarker for early diagnosis of acute myocardial infarction in humans. Eur Heart J 31: 659-666, 2010.

27. Donaldson A, Natanek SA, Lewis A, et al: Increased skeletal muscle-specific microRNA in the blood of patients with COPD. Thorax 68: 1140-1149, 2013.

28. National Lung Screening Trial Research Team; Aberle DR, Adams AM, Berg CD, et al: Reduced lung-cancer mortality with low-dose computed tomographic screening. N Engl J Med 365: 395-409, 2011.

29. Infante M, Cavuto S, Lutman FR, et al: A randomized study of lung cancer screening with spiral computed tomography: three-year results from the DANTE trial. Am J Respir Crit Care Med 180: 445-453, 2009.

30. Shen J, Liu Z, Todd NW, et al: Diagnosis of lung cancer in individuals with solitary pulmonary nodules by plasma microRNA biomarkers. BMC Cancer 11: 374, 2011.

31. Cazzoli R, Buttitta F, Di Nicola M, et al: microRNAs derived from circulating exosomes as noninvasive biomarkers for screening and diagnosing lung cancer. J Thorac Oncol 8: 1156-1162, 2013. 